\title{
Phosphine-Free Palladium Acetate-Catalyzed Suzuki Reaction in Water
}

\author{
Leifang Liu, Yuhong Zhang*, Yanguang Wang \\ Department of Chemistry, Zhejiang University, Hangzhou 310027, P.R. China. \\ Fax: +86-571-87951895; Tel: +86-571-87953253; E-mail: yhzhang@ zjuem.zju.edu.cn
}

\section{Supporting Information}

\section{Content}

General procedure for Suzuki reaction..........................S2

Characterization data of the product of the Suzuki reaction..............S3

References...................................................... 24 
General Starting materials and solvents were purchased from common commercial sources and were used without additional purification. The gas chromatography analysis was performed on a GC instrument. ${ }^{1} \mathrm{H}$ NMR spectra were recorded at $500 \mathrm{MHz}$, using TMS as internal standard. Mass spectroscopy data of the product of Suzuki reaction was collected on a MS-EI instrument.

General procedure for Suzuki reaction: A mixture of $\mathrm{Na}_{2} \mathrm{CO}_{3}(0.212 \mathrm{~g}, 2 \mathrm{mmol}), \mathrm{Pd}(\mathrm{OAc})_{2}(2 \mathrm{mg}, 1$ mol\%), PEG 2000 (3.5 g), and water $(3 \mathrm{~g})$ was heated to $50{ }^{\circ} \mathrm{C}$ with stirring. Afterward, aryl halides (1 $\mathrm{mmol})$ and arylboronic acid $(1.5 \mathrm{mmol})$ was added in the solution and the reaction carried out at $50{ }^{\circ} \mathrm{C}$ for the indicated time. After the reaction, the reaction solution was cooled to room temperature and the resulted suspend was extracted four times with diethyl ether $(4 \times 15 \mathrm{ml})$. The residue of the extraction was subjected to a second run of the Suzuki reaction by charging with the same substrates (bromotoluene, phenylboronic acid, water and $\mathrm{Na}_{2} \mathrm{CO}_{3}$ ) without further addition of $\mathrm{Pd}(\mathrm{OAc})_{2}$ or PEG. The combined ether phase was analyzed by GC/MS and then concentrated. The further purification of the product was achieved by flash chromatography on a silica gel column. 


\section{Characterization data of the product of the Suzuki reaction}

(1)

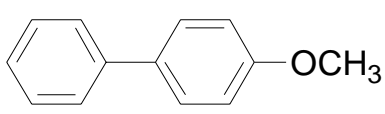

4-Methoxy-biphenyl [613-37-6, ref 1, T2-1]

${ }^{1} \mathrm{H}$ NMR (500 MHz, $\mathrm{CDCl}_{3}$, TMS): 87.54 (q, $\left.4 \mathrm{H}, J=6.7 \mathrm{~Hz}\right), 7.42(\mathrm{t}, 2 \mathrm{H}, J=7.7 \mathrm{~Hz}), 7.28(\mathrm{t}, 1 \mathrm{H}, J$ $=14.8 \mathrm{~Hz}), 6.98(\mathrm{~d}, 2 \mathrm{H}, J=4.3 \mathrm{~Hz}), 3.86(\mathrm{~s}, 3 \mathrm{H}) . \mathrm{MS}(\mathrm{EI}): \mathrm{m} / \mathrm{z}(\%): 184(100)\left[\mathrm{M}^{+}\right], 169(44), 141$ (38), 115 (26), 63 (4).
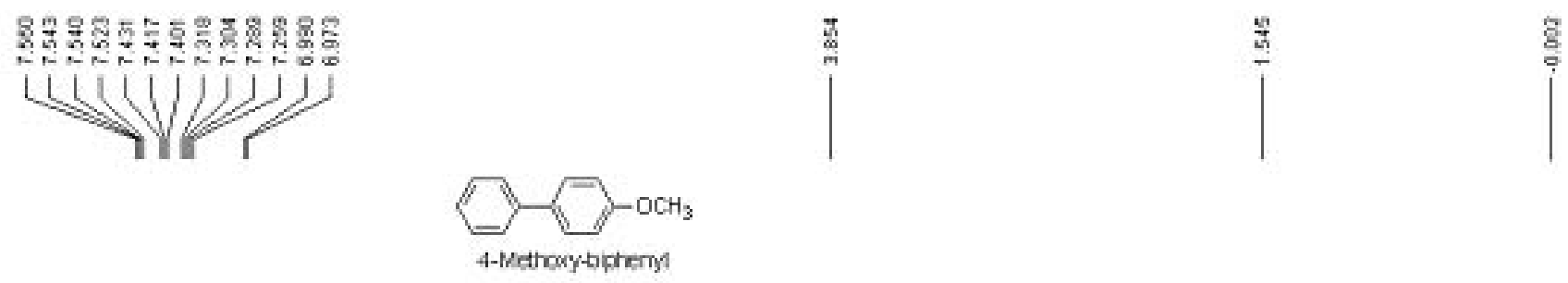

AVANCE DKXEOO Dhsjiang Uniwsrsity.

ONP $5 \mathrm{~mm}$ sample:LLF:SS in CDCL 3

Jas 10 16:21:18 2005 Pacife Standard T

USER: root

Complex_Poircs $=32768$

Spectrameter Froquency $=900.13000 \mathrm{~S} \mathrm{taHz}$

Smeep_Width $=12531.33 \mathrm{~Hz}$

Ofsel $=3065.79 \mathrm{~Hz}$

Domain = Frequency

Scans $=16$

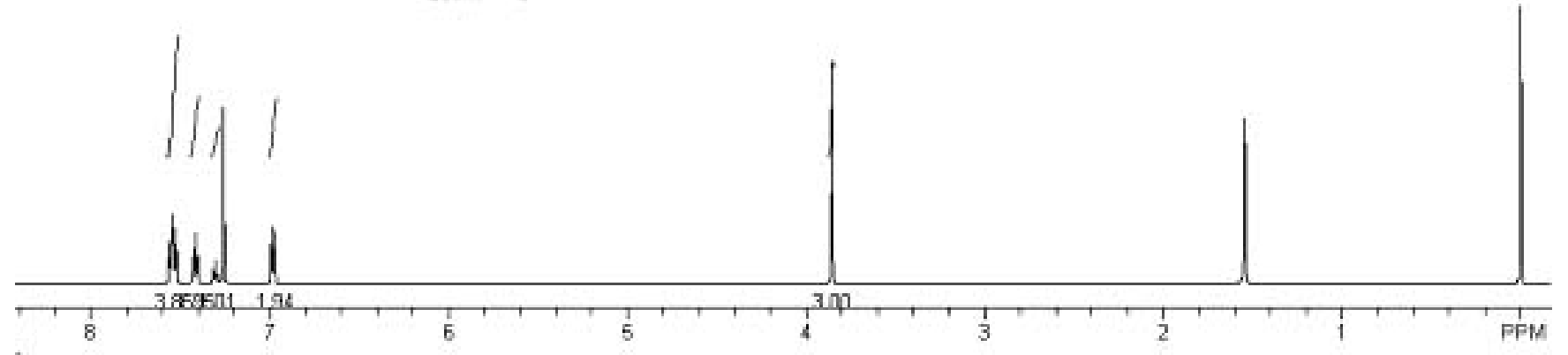


(2)

$\mathrm{HO}$

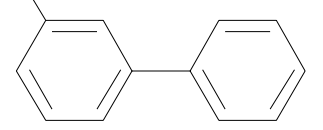

Biphenyl-3-ol [580-51-8, ref 2, T2-2]

${ }^{1} \mathrm{H}$ NMR (500 MHz, CDCl 3 , TMS): $\delta 7.56$ (d, $2 \mathrm{H}, J=7.7 \mathrm{~Hz}$ ), 7.43 (t, $2 \mathrm{H}, J=7.6 \mathrm{~Hz}$ ), $\delta 7.34$ (m, 2 H), 7.17 (d, $1 \mathrm{H}, J=7.7 \mathrm{~Hz}), 7.06$ (s, $1 \mathrm{H}), 6.82$ (m, $1 \mathrm{H}$ ) ,4.82 (s, $1 \mathrm{H}) . \mathrm{MS}$ (EI): m/z (\%): 170 (100) $\left[\mathrm{M}^{+}\right], 141(20), 115(14)$.
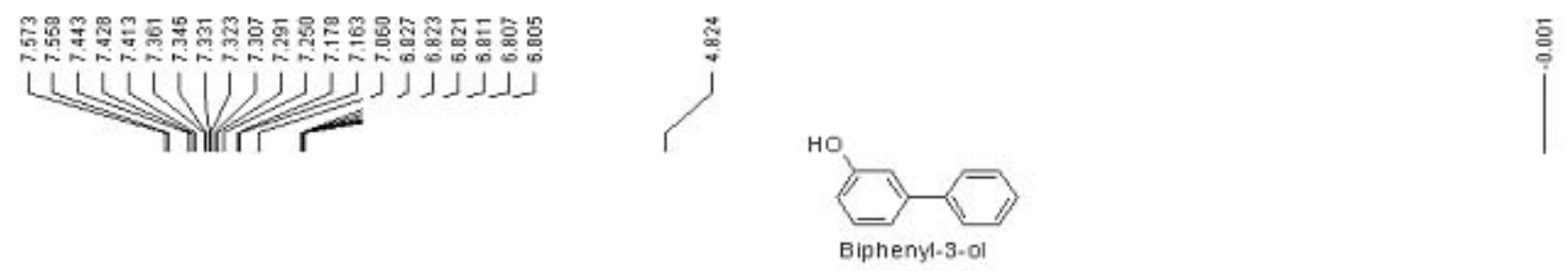

AVANCE DMDSO0 Zhejiang University, ONP $5 \mathrm{~mm}$ sample1: $\mathrm{F}$. OH in $\mathrm{CDCL} 3$ Apr 4 16:32:39 2005 Pacific Daylight

USER: root

Complex_Points $=32768$

Spectrometer_Frequency $=500,130005 \mathrm{MHz}$

Sweep_Width $=12531.33 \mathrm{~Hz}$

Offset $=2480.03 \mathrm{~Hz}$

Domain $=$ Frequency

Scans $=16$

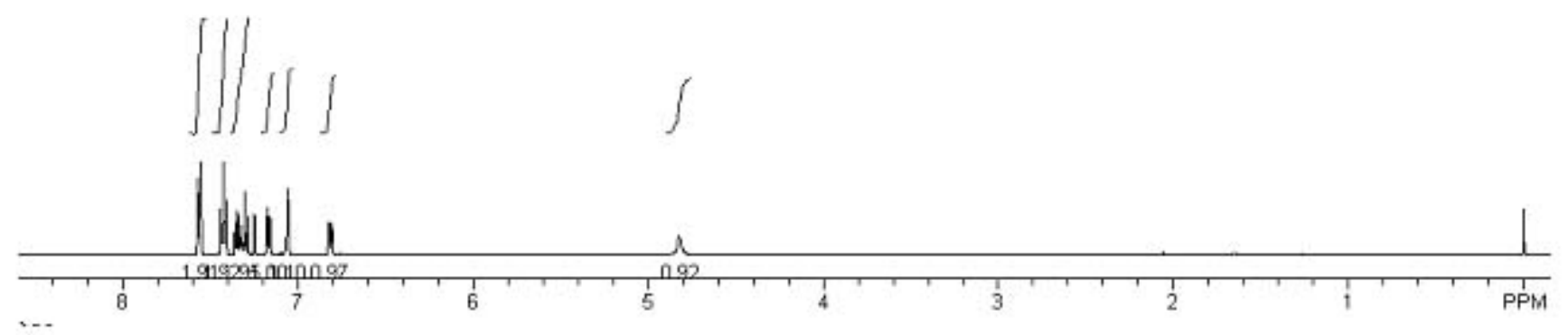


(3)

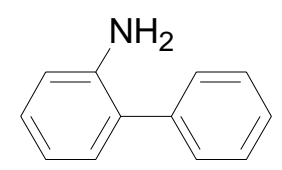

Biphenyl-2-yiamine [90-41-5, ref 3, T2-3]

${ }^{1} \mathrm{H}$ NMR (500 MHz, CDCl, TMS): 87.44 (m, 4 H), 7.34 (m, 1 H), 7.15 (m, 2 H), 6.83 (m, $\left.1 \mathrm{H}\right), 6.76$

(d, $1 \mathrm{H}, J=7.8 \mathrm{~Hz}$ ), 3.73 (s, $2 \mathrm{H})$. MS (EI): m/z (\%): 169 (99) [M+'], 168 (100), 167 (47), 139 (9), 115(14), 84 (20), 63 (14), 51 (17), 39 (20).
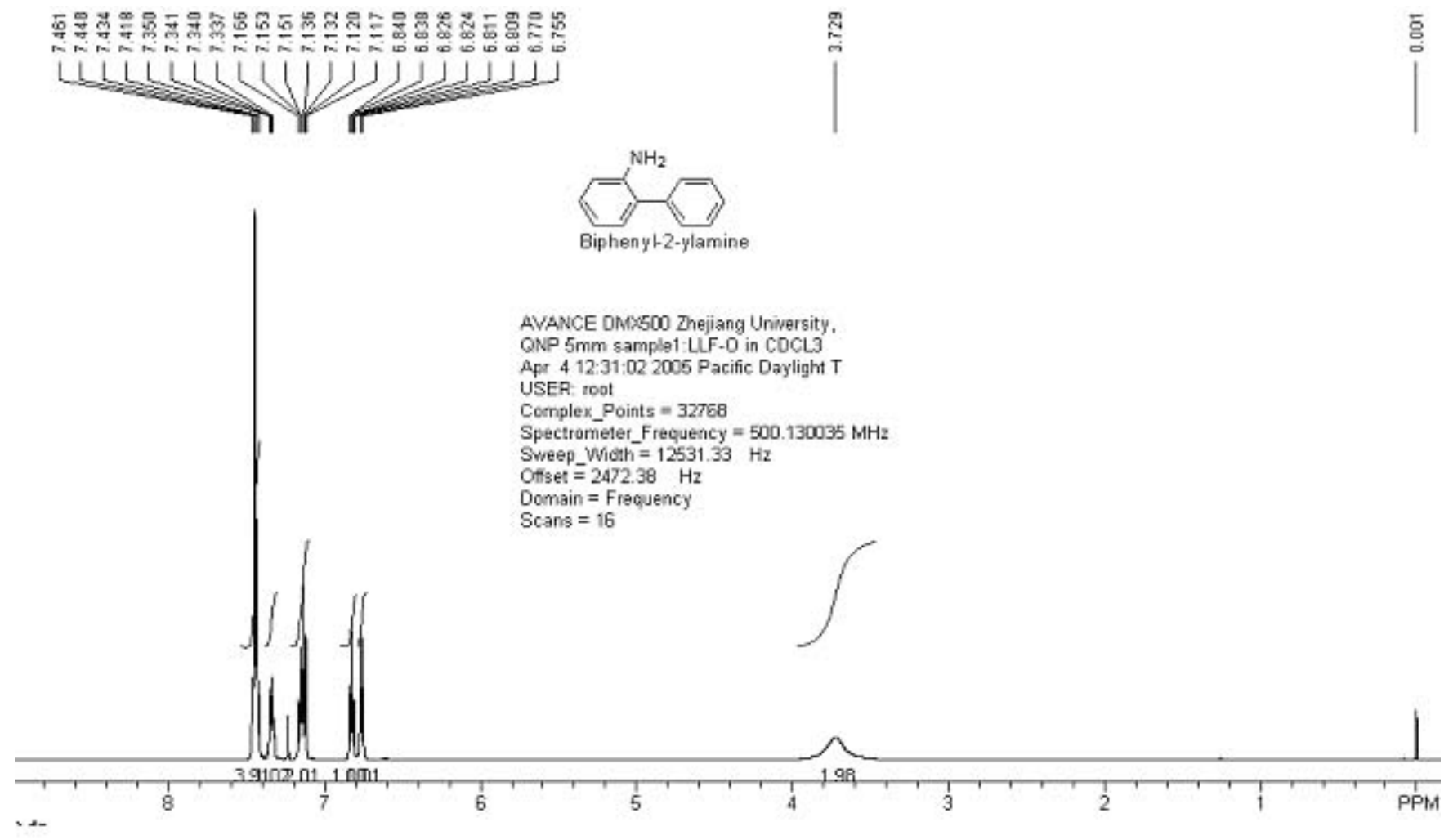
(4)

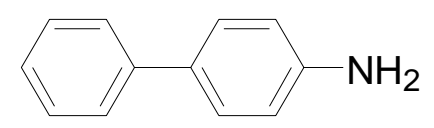

Biphenyl-4-ylamine [92-67-1, ref 4, T2-4]

${ }^{1} \mathrm{H}$ NMR (500 MHz, CDCl 3 , TMS): $\delta 7.53$ (d, $\left.2 \mathrm{H}, J=8.0 \mathrm{~Hz}\right), 7.39$ (m, $4 \mathrm{H}$ ), 7.15 (m, $2 \mathrm{H}$ ), 7.26 (t, 1 $\mathrm{H}, J=7.8 \mathrm{~Hz}), 6.76(\mathrm{~d}, 1 \mathrm{H}, J=8.0 \mathrm{~Hz}), 6.75(\mathrm{t}, 2 \mathrm{H}, J=8.5 \mathrm{~Hz}), 3.70(\mathrm{~s}, 2 \mathrm{H}) . \mathrm{MS}(\mathrm{EI}): \mathrm{m} / \mathrm{z}(\%): 169$ (100) $\left[\mathrm{M}^{+}\right], 168(20), 167(11), 141(7), 115(7), 83(6)$.
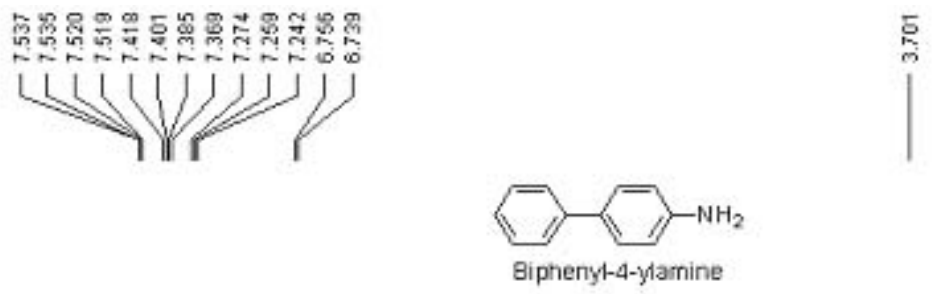

AVANCE DMOEOD Zhejiang University.

ONP $5 \mathrm{~mm}$ sample1: LLF.P in CDCL 3 .

Apr 6 15:03:12 2005 Pacific Daylight T

USER: root

Complex_Points $=32768$

Spectrometer_Frequency $=500.130035 \mathrm{MHz}$

Sweep_Wvidth $=12531.33 \mathrm{~Hz}$

Ollsel $=247697 \quad \mathrm{~Hz}$

$\int$

Domain $=$ Frequency

Scans $=16$

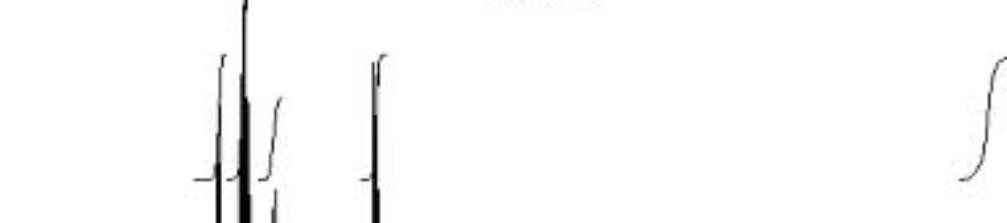


(5)

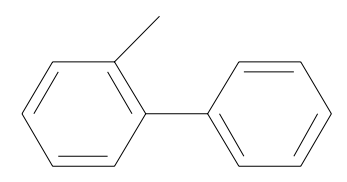

2-Methyl-biphenyl [643-58-3, ref 4, T2-5]

${ }^{1} \mathrm{H}$ NMR (500 MHz, CDCl 3 , TMS): $\delta 7.42$ (t, $2 \mathrm{H}, J=7.5 \mathrm{~Hz}$ ), 7.34 (m, $\left.3 \mathrm{H}\right), 7.26$ (m, $4 \mathrm{H}$ ), 2.28 (s, 3

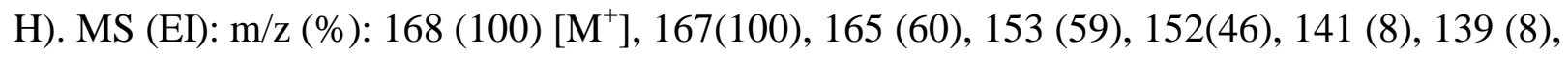
115(14), 83(14), 63(6), 51(4).
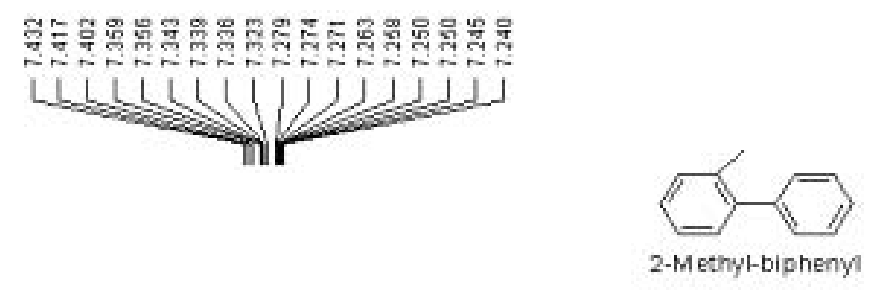

AVANCE OWSOD Znejiang University. DNP $5 \mathrm{~mm}$ sampla: LF. 36 in CDCL3 Jan 10 15:16:41 2005 Pacisc Standard T USER: root

Complex_Point $s=32768$

Spectrometer_Frequency $=500,130005 \mathrm{MHz}$

Sweep_width $=12531.33 \mathrm{~Hz}$

Oftse1 $=3005.79 \mathrm{~Hz}$

11

Domain = Frequency

Scans $=16$
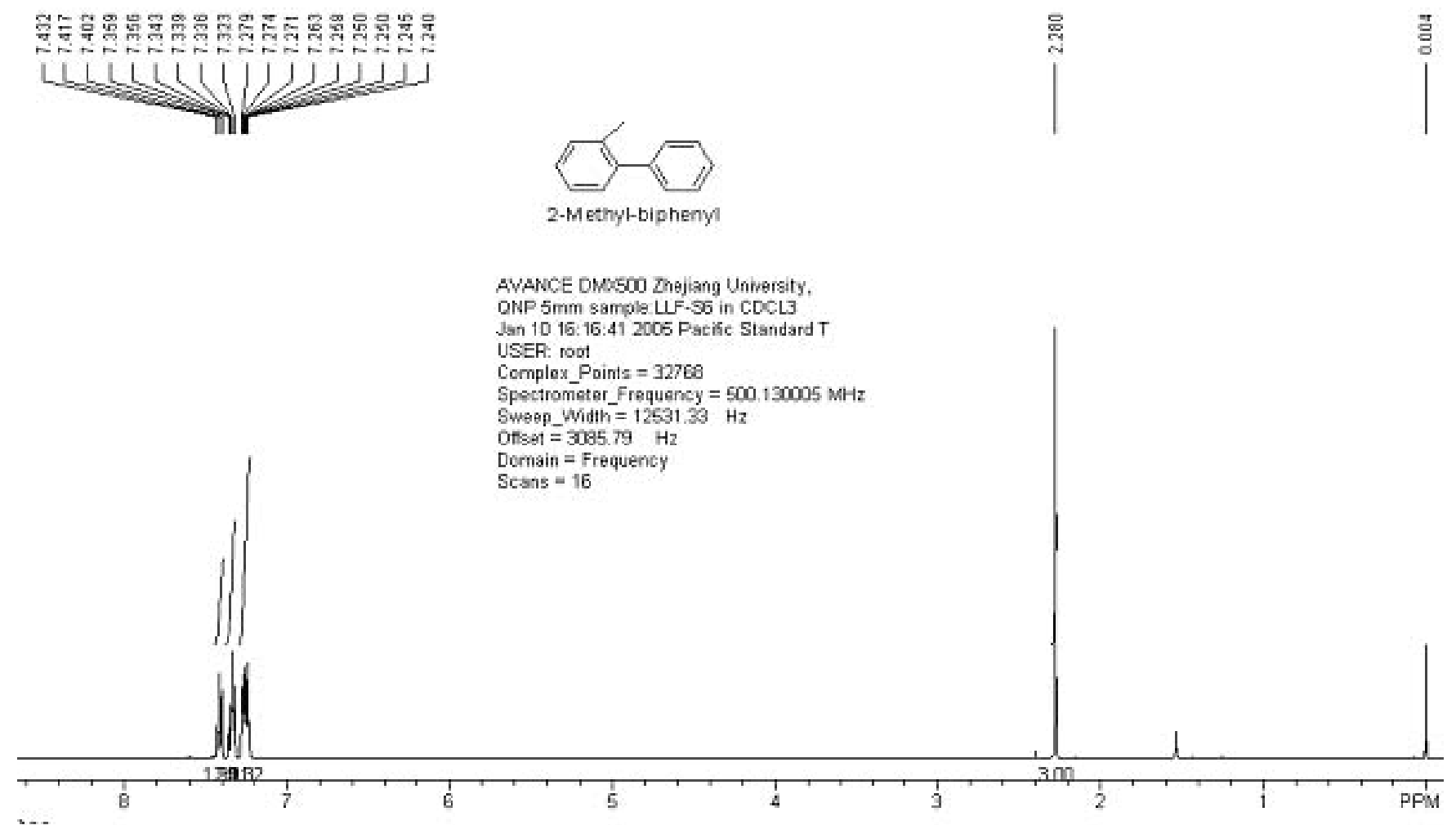
(6)

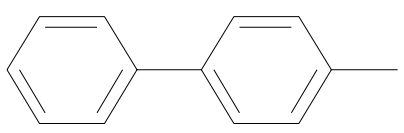

4-Methyl-biphenyl [644-08-6, ref 1,T2-6]

${ }^{1} \mathrm{H}$ NMR (500 MHz, $\left.\mathrm{CDCl}_{3}, \mathrm{TMS}\right): \delta 7.58$ (d, $\left.2 \mathrm{H}, J=7.5 \mathrm{~Hz}\right), 7.49$ (d, $\left.2 \mathrm{H}, J=8.0 \mathrm{~Hz}\right), 7.43(\mathrm{t}, 2 \mathrm{H}, J$ $=7.5 \mathrm{~Hz}), 7.32(\mathrm{t}, 1 \mathrm{H}, J=7.3 \mathrm{~Hz}), 7.25(\mathrm{~d}, 2 \mathrm{H}, J=7.5 \mathrm{~Hz}) . \mathrm{MS}(\mathrm{EI}): \mathrm{m} / \mathrm{z}(\%): 168(100)\left[\mathrm{M}^{+}\right]$, 167(68), 165 (22), $152(20), 115(6)$.
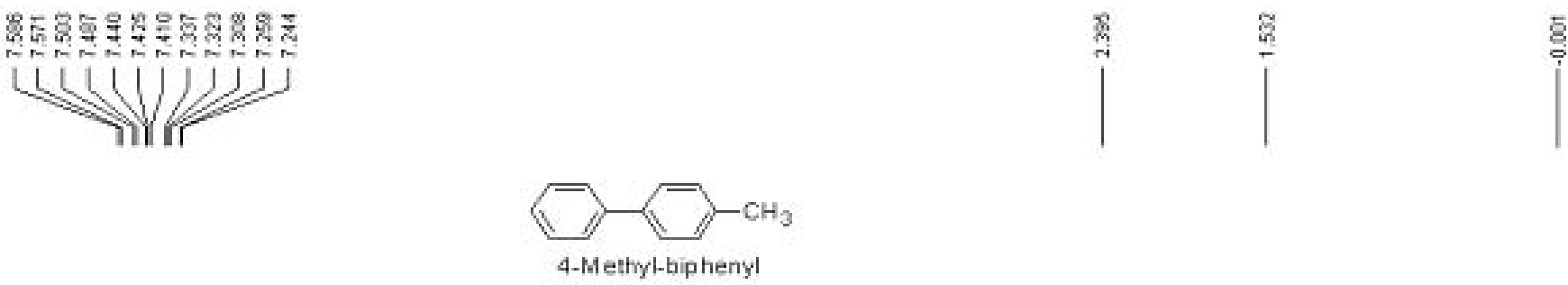

AVANCE DMXCO0 Dnsjiang Univeraity.

ONP $5 \mathrm{~mm}$ sample1:LLF 3.19 in CDCLS

Mar 18 15.52:12 2005 Pacific Standard T

USER: root

Complex_Parts $=32768$

Spectrometer_Frequency $=500130035 \mathrm{MHz}$

Swose Width $=12531.30 \mathrm{~Hz}$

Ofset $=3479.69 \mathrm{~Hz}$

Domain = Frequenty

Scans $=16$
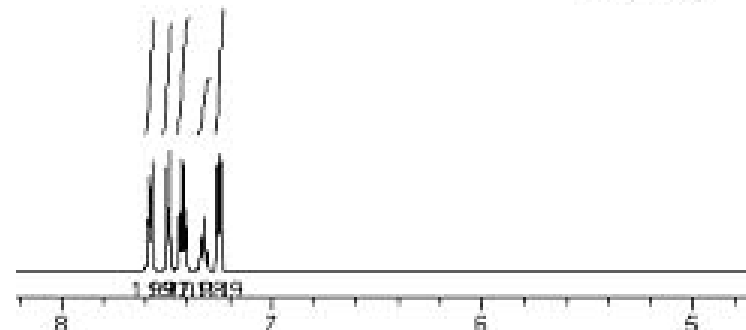
(7)

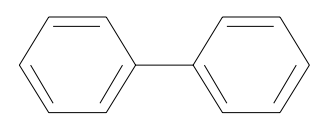

Biphenyl [92-52-4, ref 9, T2-7]

${ }^{1} \mathrm{H}$ NMR (500 MHz, $\mathrm{CDCl}_{3}$, TMS): $\delta 7.60(\mathrm{~d}, 4 \mathrm{H}, J=7.5 \mathrm{~Hz}), 7.44(\mathrm{t}, 4 \mathrm{H}, J=7.2 \mathrm{~Hz}), 7.35(\mathrm{t}, 2 \mathrm{H}, J$

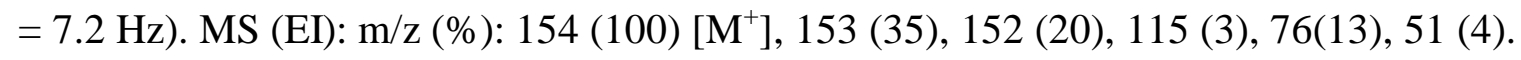
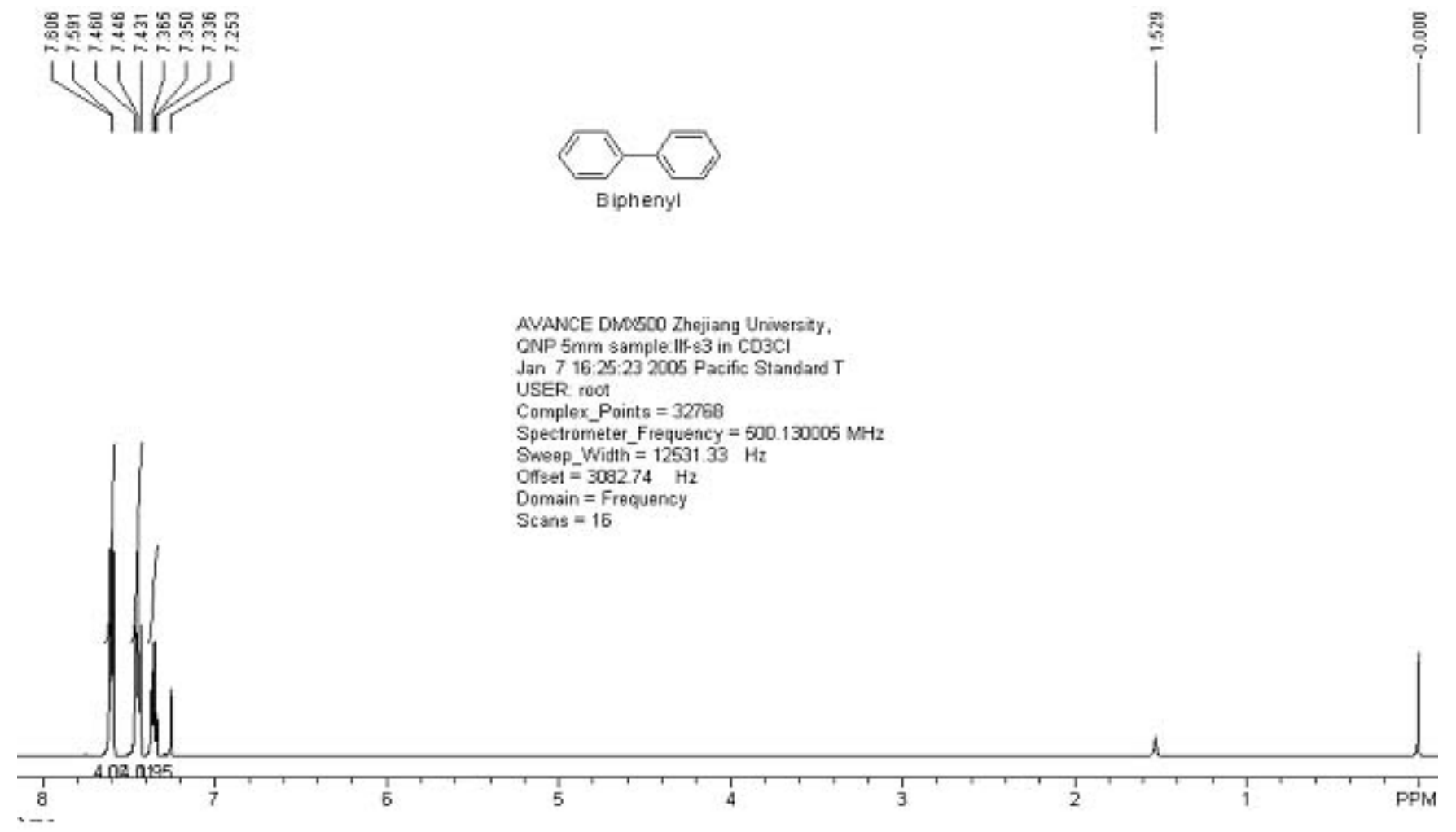

AVANCE DHAXSCO Zhejiang University,

CNP $5 \mathrm{~mm}$ sample. IlF 83 in $\mathrm{CO} 3 \mathrm{Cl}$

Jan 7 16:25:23 2005 Pacific Standard T

USER root

Complex Points = 3278

Spectrometer_Frequency $=600.130005 \mathrm{MH}$

Sweap Width $=12531.33 \mathrm{~Hz}$

I $=306274$ Hz

Domain $=$ Frequency 
(8)

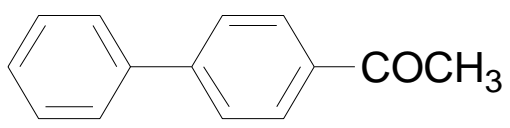

1-Biphenyl-4-yl-ethanone [92-91-1, ref 4, T2-8]

${ }^{1} \mathrm{H}$ NMR (500 MHz, $\mathrm{CDCl}_{3}$, TMS): $\delta 8.03$ (d, $2 \mathrm{H}, J=8.4 \mathrm{~Hz}$ ), 7.69 (d, $\left.2 \mathrm{H}, J=4.0 \mathrm{~Hz}\right), 7.63(\mathrm{t}, 2 \mathrm{H}, J$ $=4.5 \mathrm{~Hz}$ ), 7.48 (t, $2 \mathrm{H}, J=7.5 \mathrm{~Hz}), 7.40(\mathrm{t}, 1 \mathrm{H} \square J=7.0 \mathrm{~Hz}), 2.64$ (s, $3 \mathrm{H}) . \mathrm{MS}(\mathrm{EI}): \mathrm{m} / \mathrm{z}(\%): 196(51)$ $\left[\mathrm{M}^{+}\right], 181(100), 153(33), 152(51), 76(13), 43(4)$.
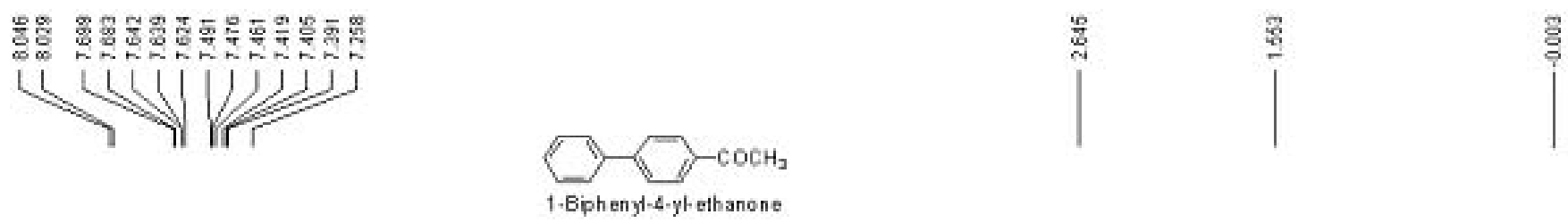

AVANUCE DMKSOO Zhejiang University,

ONP $5 \mathrm{~mm}$ sample:LLF-S4 in COCL3

Jan 10 16:25:48 2005 Pacific Standard T

USER root

Complex_Points $=32758$

Spectrometer_Frequency $=500.130005 \mathrm{MHz}$

Sweep_Width $=12531.33 \mathrm{~Hz}$

Offet $=3095.79 \mathrm{~Hz}$

Domain = Frequency

Scans $=16$
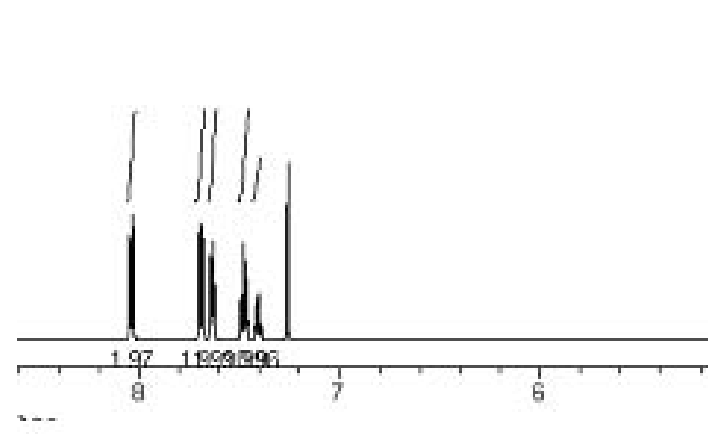
(9)

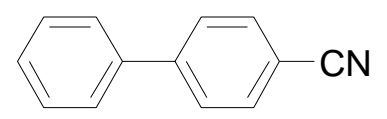

Biphenyl-4-carbonitrile [2920-38-9, ref 1, T2-9]

${ }^{1} \mathrm{H}$ NMR (500 MHz, $\mathrm{CDCl}_{3}$, TMS): $\delta 7.73$ (d, $\left.2 \mathrm{H}, J=8.5 \mathrm{~Hz}\right), 7.68$ (d, $\left.2 \mathrm{H}, J=8.5 \mathrm{~Hz}\right), 7.59$ (t, $2 \mathrm{H}, J$ $=4.5 \mathrm{~Hz}), 7.48(\mathrm{t}, 2 \mathrm{H}, J=7.5 \mathrm{~Hz}), 7.42$ (m, $1 \mathrm{H}) . \mathrm{MS}(\mathrm{EI}): \mathrm{m} / \mathrm{z}(\%): 179(100)\left[\mathrm{M}^{+}\right], 151(16), 89(10)$, $76(14)$.
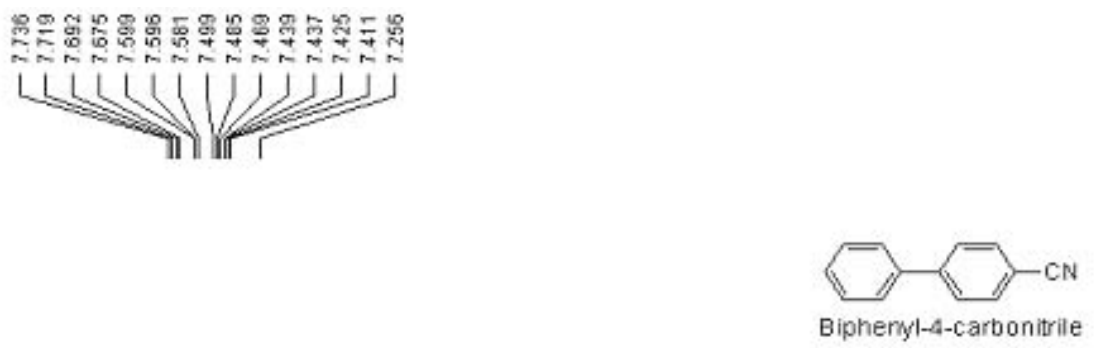

AVANCE DMXE00 Zhejiang Unimersity, ONP $5 \mathrm{~mm}$ sample1:LLF.CN in CDCL3 Aor 4 12:27:11 2005 Pacific Daylight USER: toot

Complex_Points $=32768$

Spectrometer_Frequency $=500.130005 \mathrm{MHz}$

Sweep_Width $=12531.33 \mathrm{~Hz}$

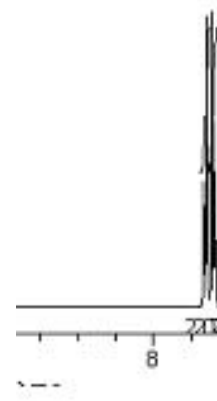

Offset $=2485.77 \quad \mathrm{~Hz}_{2}$

Domain $=$ Frecuency

Seans $=16$ 


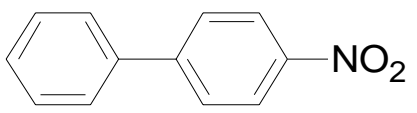

4-Nitro-biphenyl [92-93-3, ref 1, T2-10]

${ }^{1} \mathrm{H}$ NMR (500 MHz, $\mathrm{CDCl}_{3}$, TMS): $\delta 8.32(\mathrm{~d}, 2 \mathrm{H}, J=9.0 \mathrm{~Hz}), 7.75(\mathrm{~d}, 2 \mathrm{H}, J=9.0 \mathrm{~Hz}), 7.64(\mathrm{~d}, 2 \mathrm{H}, J$ $=7.0 \mathrm{~Hz}), 7.51(\mathrm{t}, 2 \mathrm{H}, J=7.5 \mathrm{~Hz}), 7.46(\mathrm{t}, 1 \mathrm{H}, J=7.2 \mathrm{~Hz}) . \mathrm{MS}(\mathrm{EI}): \mathrm{m} / \mathrm{z}(\%): 199(100)\left[\mathrm{M}^{+}\right]$, 169(37), 152 (100), 141 (24), 115 (13), 76 (13), 63 (7), 51(6).
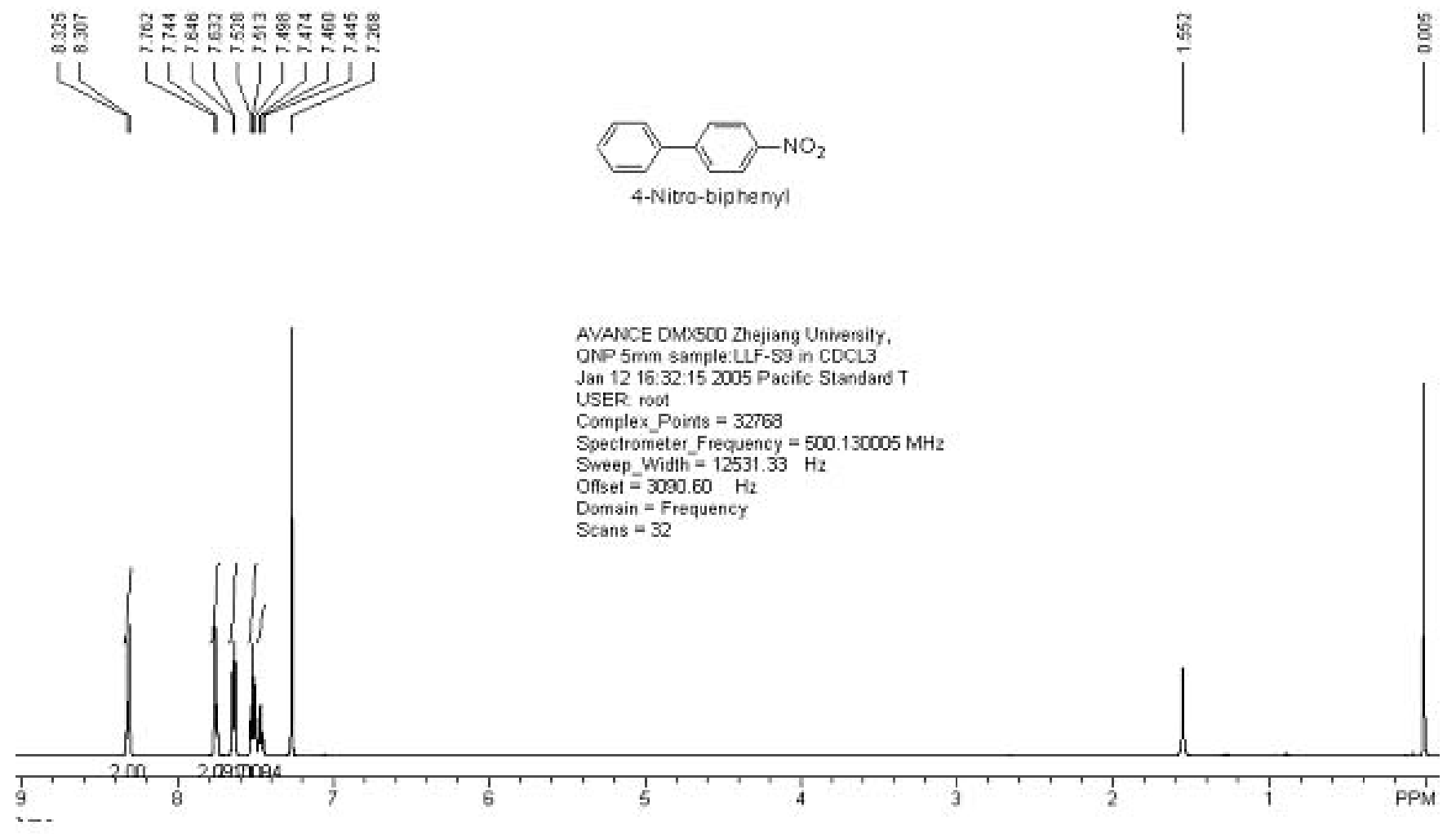
(11)

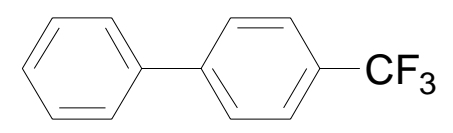

4-Trifluoromethyl-biphenyl [398-36-7, ref 9, T3-2]

${ }^{1} \mathrm{H} \mathrm{NMR}\left(500 \mathrm{MHz}, \mathrm{CDCl}_{3}\right.$, TMS): $\delta 7.69$ (s, $\left.4 \mathrm{H}\right), 7.60$ (d, $\left.2 \mathrm{H}, J=8.0 \mathrm{~Hz}\right), 7.48$ (t, $2 \mathrm{H}, J=7.5 \mathrm{~Hz}$ ), 7.41 (t, $1 \mathrm{H}, J=7.3 \mathrm{~Hz}), \mathrm{MS}(\mathrm{EI}): \mathrm{m} / \mathrm{z}(\%): 222(100)\left[\mathrm{M}^{+}\right], 201$ (13), 152 (26), 86(4).

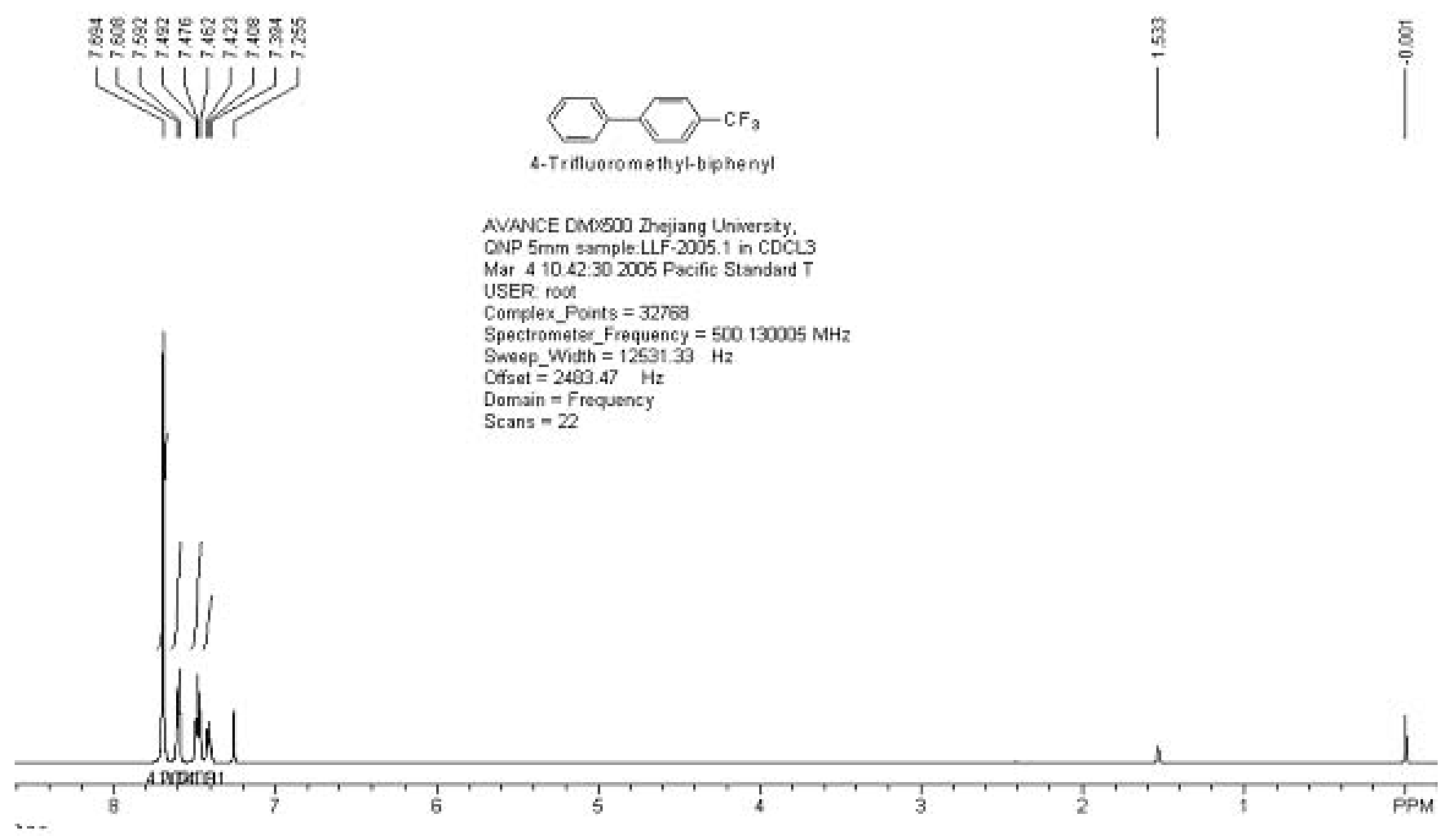


(12)

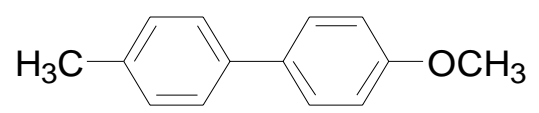

4-Methoxy-4'-methyl-biphenyl [53040-92-9, ref 9, T3-3]

${ }^{1} \mathrm{H}$ NMR (500 MHz, $\left.\mathrm{CDCl}_{3}, \mathrm{TMS}\right): \delta 7.51$ (d, $\left.2 \mathrm{H}, J=8.0 \mathrm{~Hz}\right), 7.45$ (d, $\left.2 \mathrm{H}, J=8.0 \mathrm{~Hz}\right), 7.22$ (d, $2 \mathrm{H}, J$

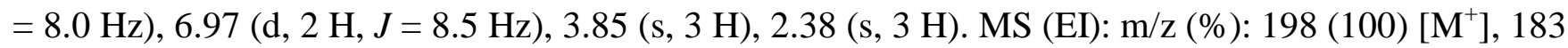
(54), 155 (27), $128(8), 115(5)$.
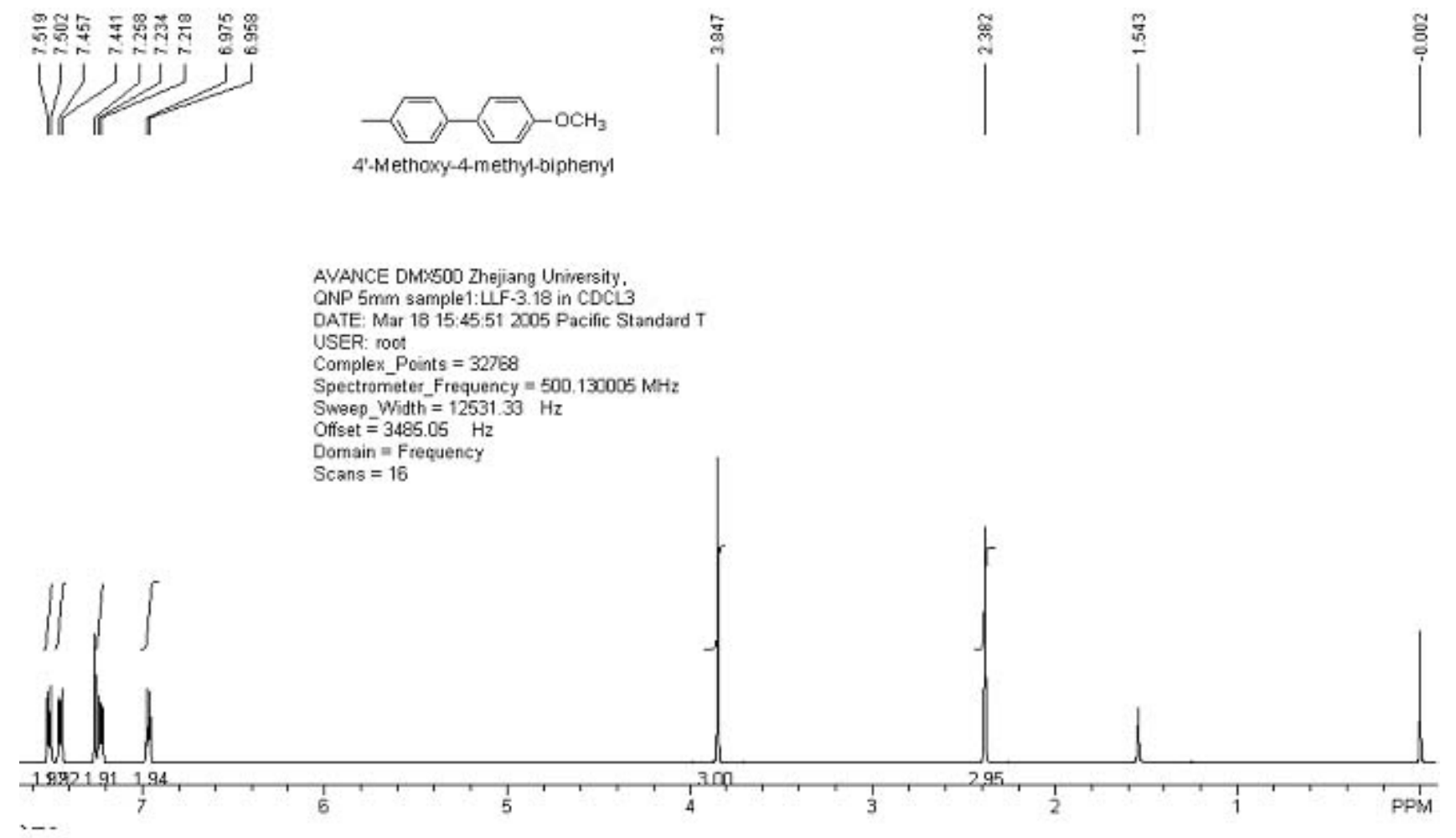
(13)

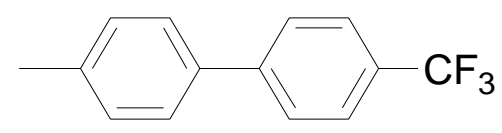

4'-Methyl-4-trifluoromethyl-biphenyl [97067-18-0, ref 5, T3-4]

${ }^{1} \mathrm{H}$ NMR (500 MHz, CDCl 3 , TMS): $\delta 7.67$ (s, $\left.4 \mathrm{H}\right), 7.50(\mathrm{~d}, 2 \mathrm{H}, J=8.1 \mathrm{~Hz}), 7.28(\mathrm{~d}, 2 \mathrm{H}, J=8.0 \mathrm{~Hz}$ ), 2.40 (s,3 H). MS (EI): m/z (\%): 236 (100) [M+1, 235 (25), 167 (38), 165(35), 152(8), 91 (7).

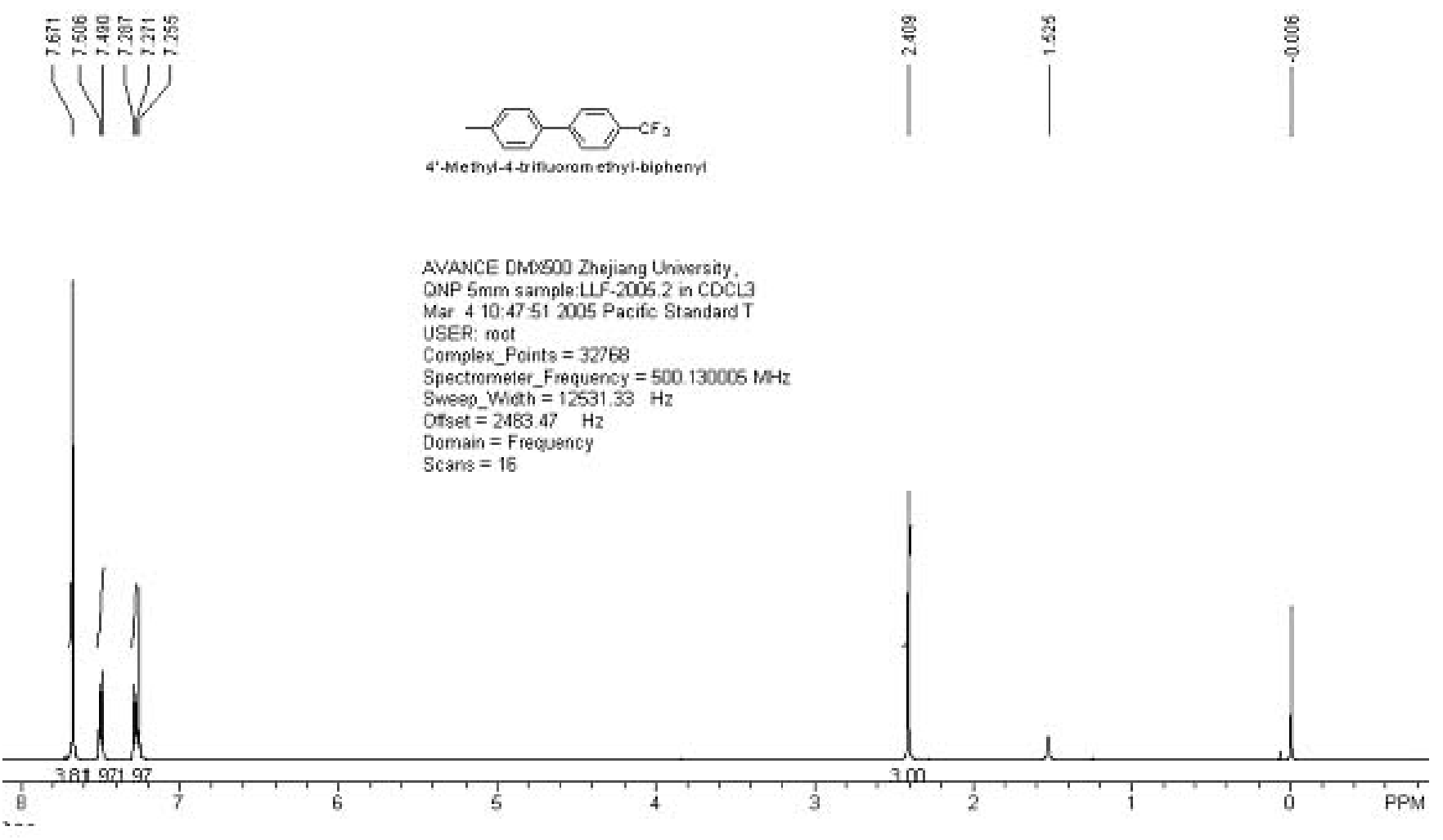


(14)

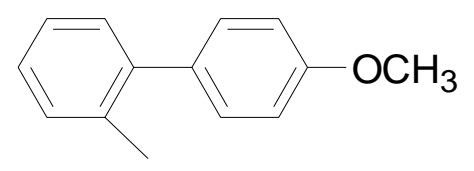

4'-Methoxy-2-methyl-biphenyl [92495-54-0, ref 1, T3-5]

${ }^{1} \mathrm{H}$ NMR (500 MHz, CDCl 3 , TMS): $\delta 7.23$ (m, 6 H), 3.84 (s, 3 H), 2.27 (s, 3 H). MS (EI): m/z (\%):198 (100) $\left[\mathrm{M}^{+}\right], 183$ (22), 167 (19), 165 (21), 155 (21), 153 (21), 128 (12), 115 (9).
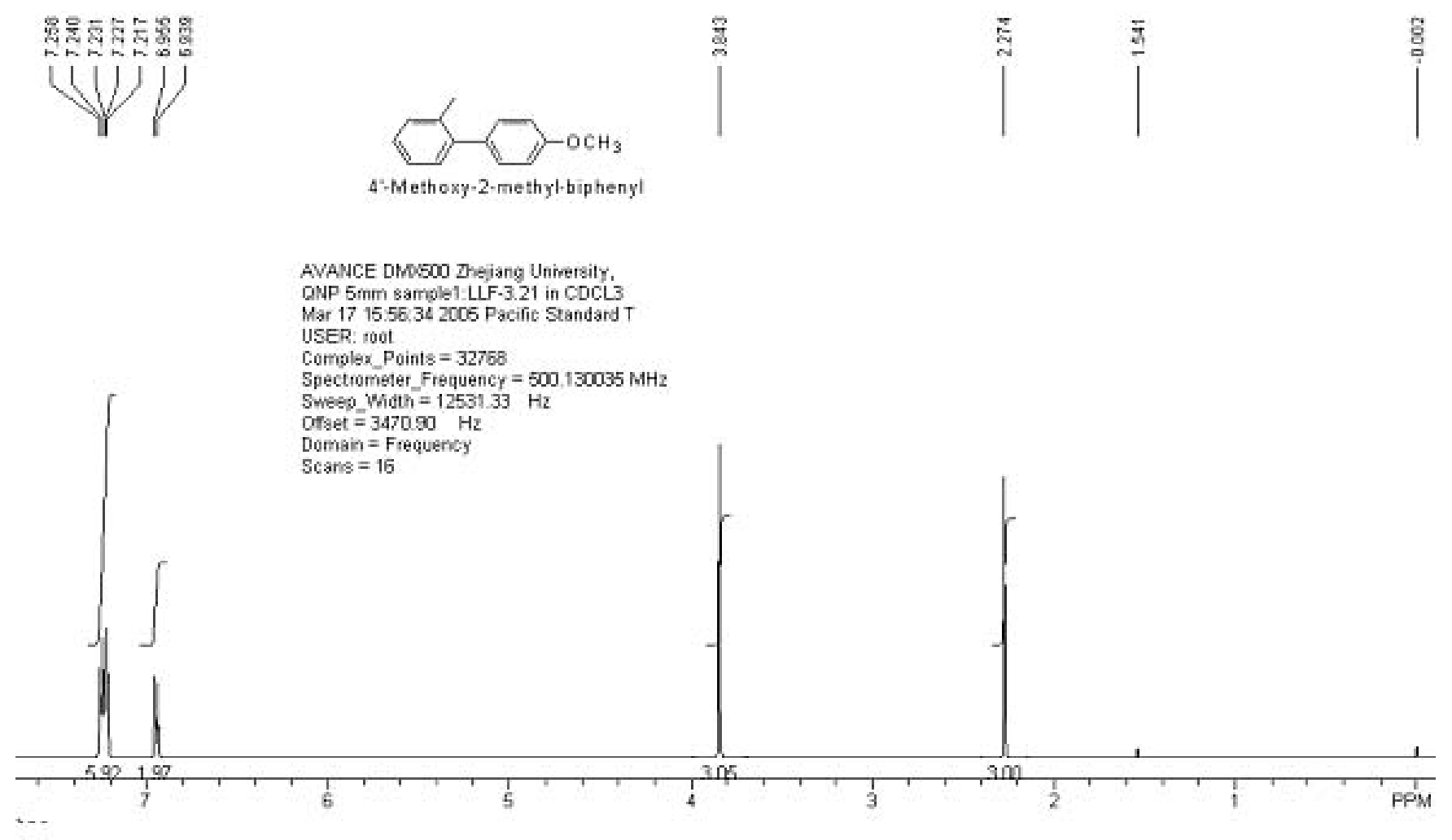


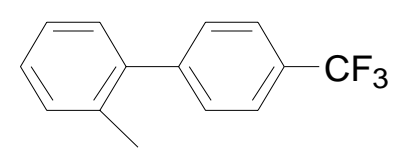

\section{2-Methyl-4'-trifluoromethyl-biphenyl [167021-49-0, ref 10, T3-6]}

${ }^{1} \mathrm{H}$ NMR (500 MHz, $\mathrm{CDCl}_{3}$, TMS): $\delta 7.70$ (d, $2 \mathrm{H}, J=8.0 \mathrm{~Hz}$ ), 7.46 (d, $\left.2 \mathrm{H}, J=8.5 \mathrm{~Hz}\right), 7.30(\mathrm{~m}, 3 \mathrm{H}$ ), 7.24 (d, 1 H, J= 7.5 Hz) 2.29 (s, 3 H). MS (EI): m/z (\%):236 (86) [M+1, 217 (11), 167 (100), 166(32), $165(61), 152(21) 82(10)$.

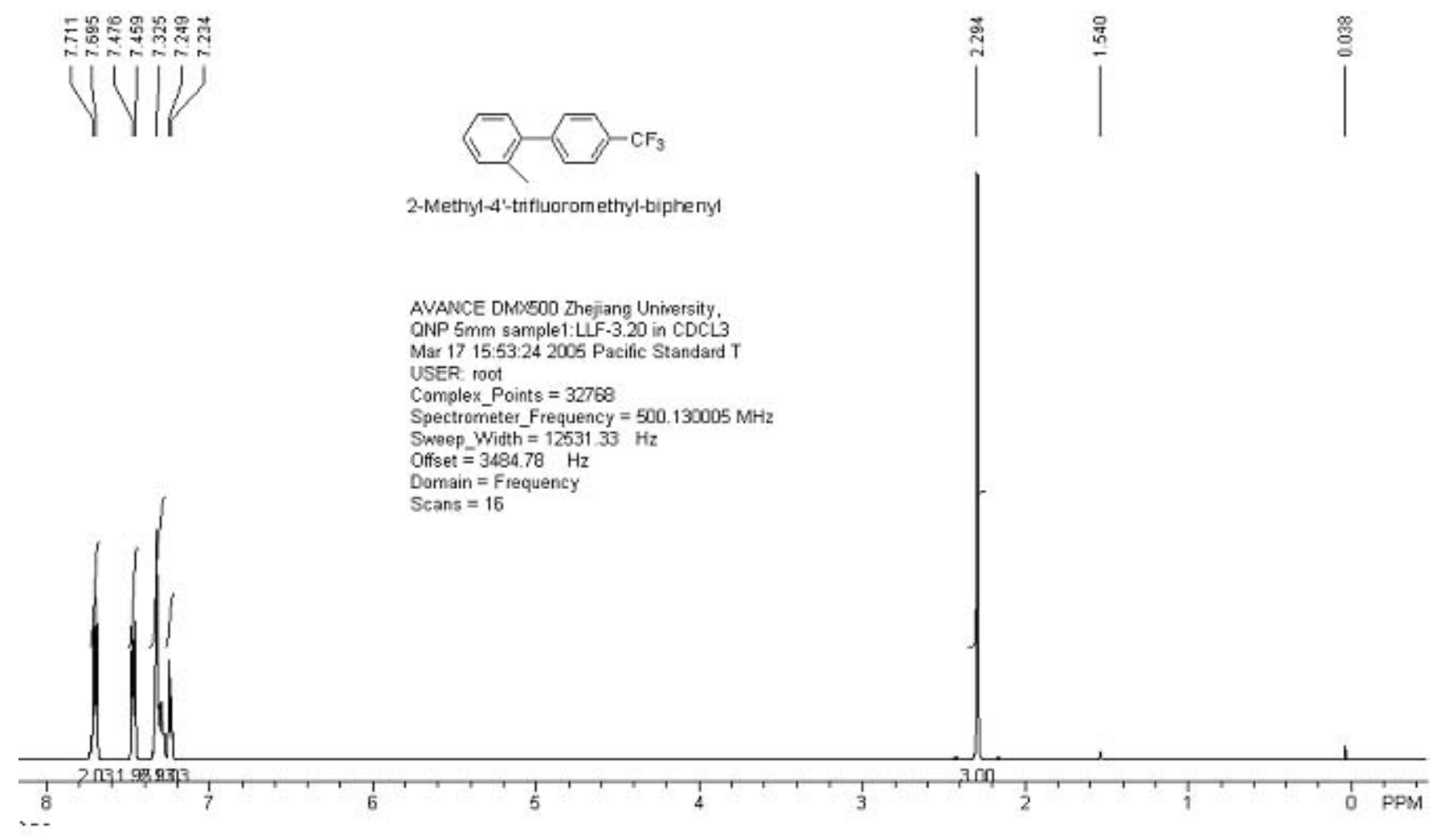


(16)

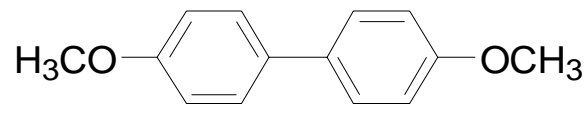

4, 4'-Dimethoxy-biphenyl [2132-80-1, ref 8, T3-7]

${ }^{1} \mathrm{H}$ NMR (500 MHz, CDCl 3 , TMS): $\delta 7.52$ (d, $4 \mathrm{H}, J=9.0 \mathrm{~Hz}$ ), 7.00 (d, $4 \mathrm{H}, J=8.5 \mathrm{~Hz}$ ), 3.89(s, $\left.6 \mathrm{H}\right)$.

MS (EI): m/z (\%): 214 (100) [M+1], 199 (81), 171 (24), 156 (8), 128 (13), 102 (16), 39 (10).
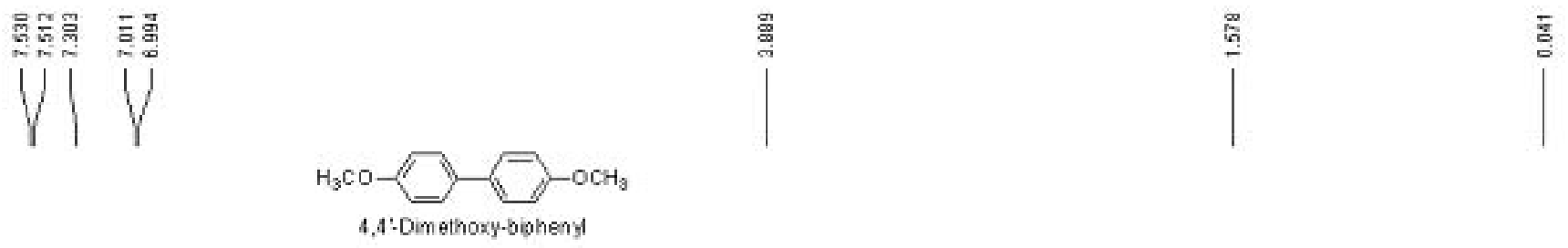

AVANCE DWNSCO Thejiang University.

CNP $5 \mathrm{~mm}$ sample:LLF.S15 in CDCB

Jan 19 16:17:55 2005 Pacific Standard T

USER; root

Complex. Points $=32768$

Spectrometer Frequency $=500.130005 \mathrm{MHz}$

Sweep_Width $=12531,30 \mathrm{~Hz}$

Otfset $=3107.97 \mathrm{~Hz}$

Domain $=$ Frequency

Scans $=16$

$\begin{array}{r}3 \\ \square \\ \hline-1\end{array}$
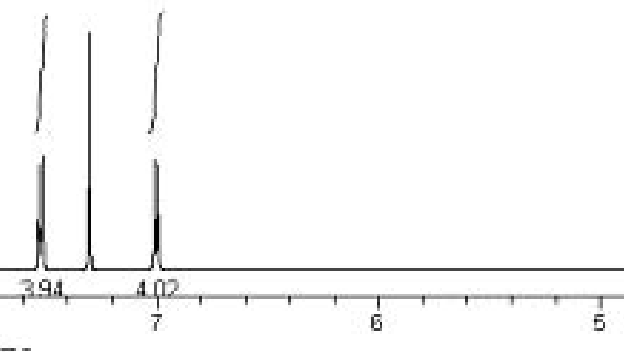

, fin 


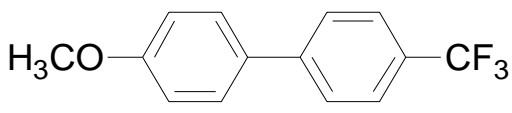

4'-Methoxy-4-trifluoromethyl-biphenyl [10355-12-1, ref 7, T3-8]

${ }^{1} \mathrm{H}$ NMR (500 MHz, $\mathrm{CDCl}_{3}$, TMS): $\delta 7.65$ (q, $\left.4 \mathrm{H}, J=6.6 \mathrm{~Hz}\right), 7.54$ (d, $\left.2 \mathrm{H}, J=8.7 \mathrm{~Hz}\right), 7.00$ (d, $2 \mathrm{H}, J$

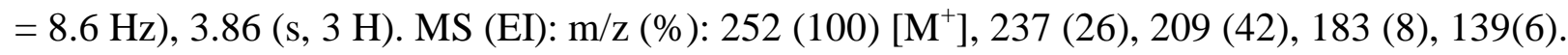

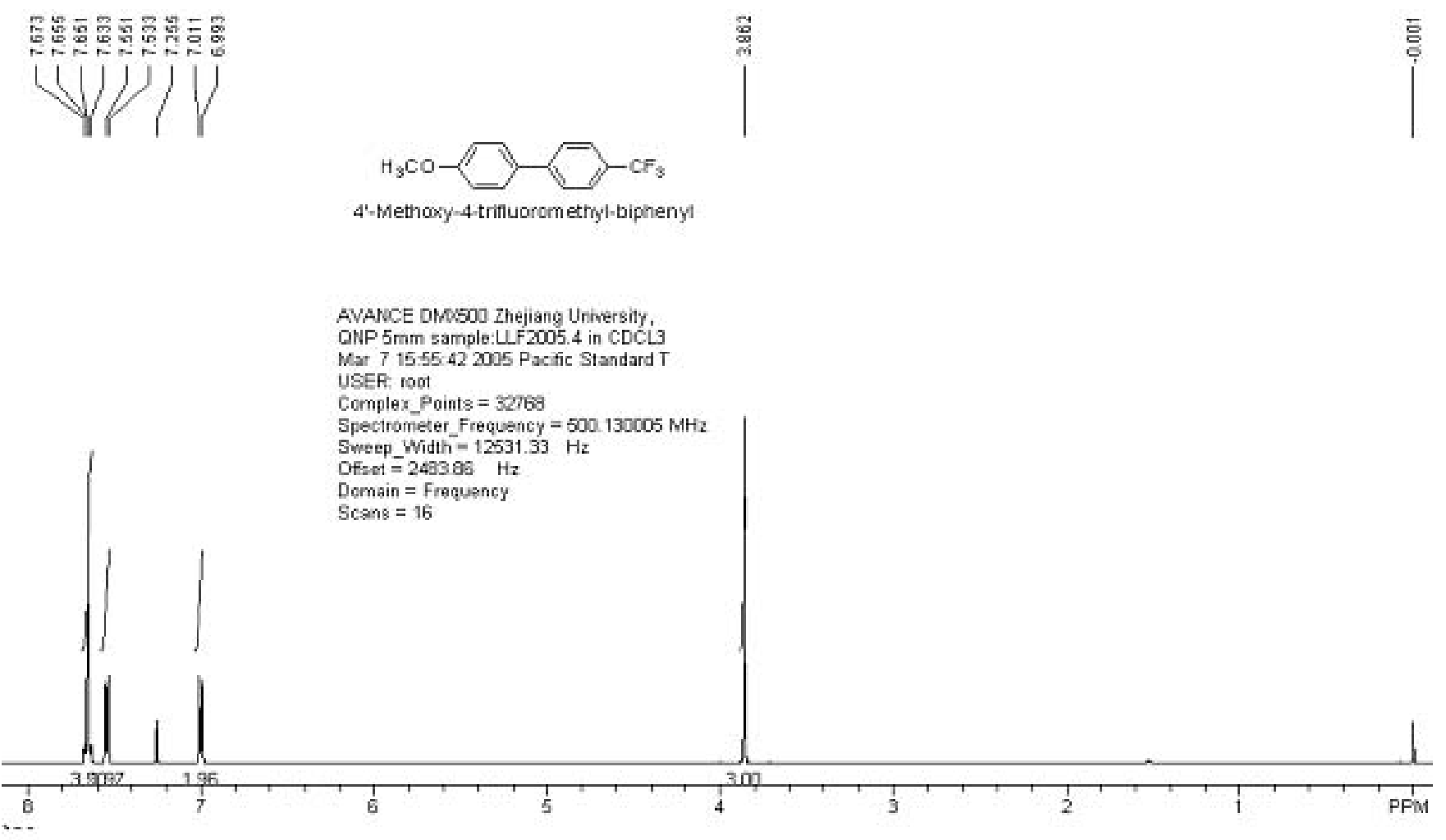




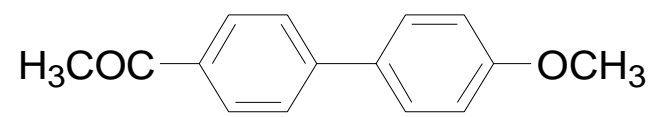

1-(4'-Methoxy-biphenyl-4-yl)-ethanone [3021-18-6, ref 6, T3-9]

${ }^{1} \mathrm{H}$ NMR (500 MHz, $\mathrm{CDCl}_{3}$, TMS): $\delta 8.00$ (d, $\left.2 \mathrm{H}, J=8.5 \mathrm{~Hz}\right), 7.65$ (d, $\left.2 \mathrm{H}, J=8.5 \mathrm{~Hz}\right), 7.58$ (d, $2 \mathrm{H}, J$ $=9.0 \mathrm{~Hz}), 7.00(\mathrm{~d}, 2 \mathrm{H}, J=8.5 \mathrm{~Hz}), 3.87$ (s, $3 \mathrm{H}), 2.61$ (s, $3 \mathrm{H}) . \mathrm{MS}(\mathrm{EI}): \mathrm{m} / \mathrm{z}(\%): 226(68)\left[\mathrm{M}^{+}\right], 211$ (100), 183 (10), 152 (10), 139 (21), 105 (6), 43 (3).
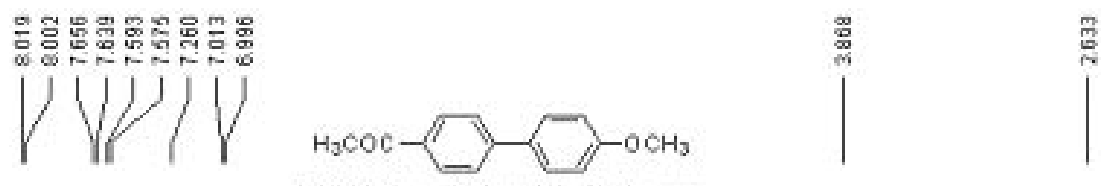

1-(4'-Methoxy-biphenyl-4-yl)-ethanone

AVANCE DMXSOO Dhejiang Unirersity. CNP $5 \mathrm{~mm}$ sample:LLF.S13 in COC13 Jan 18 15:17:36 2005 Pacíc Standard T USER: root

Complex Points $=32768$

Spectrometer_Frequency $=500.130005 \mathrm{MHz}$ Sweep_Width $=12591.39 \mathrm{~Hz}$ Otiset $=3005.18 \mathrm{~Hz}$ Domain $=$ Frequency Seans $=16$
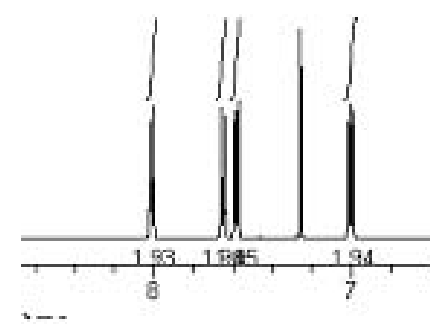


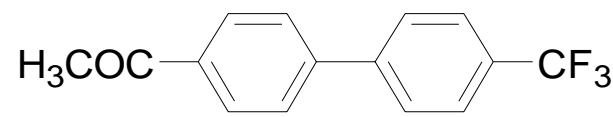

1-(4'-Trifluoromethyl-biphenyl-4-yl)-ethanone $\quad$ [142557-76-4, ref 11, T3-10]

${ }^{1} \mathrm{H}$ NMR (500 MHz, $\mathrm{CDCl}_{3}$, TMS): $\delta 8.06$ (d, $\left.2 \mathrm{H}, J=8.4 \mathrm{~Hz}\right), 7.72$ (s, $\left.4 \mathrm{H}\right), 7.69$ (d, $2 \mathrm{H}, J=8.4 \mathrm{~Hz}$ ), 2.65 (s, 3 H). MS (EI): m/z (\%): 264 (36) $\left[\mathrm{M}^{+}\right], 249$ (100), 221 (8), 201 (26), 152 (24), 43(4).
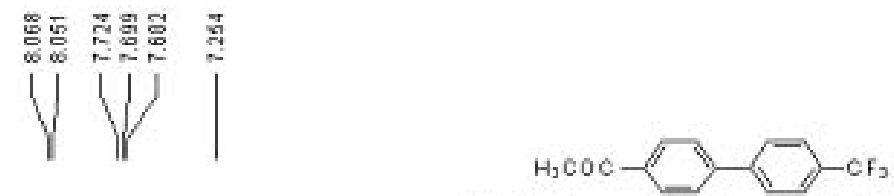

1-(4:Trifu oromethylbipheny 4-y D-ethanone

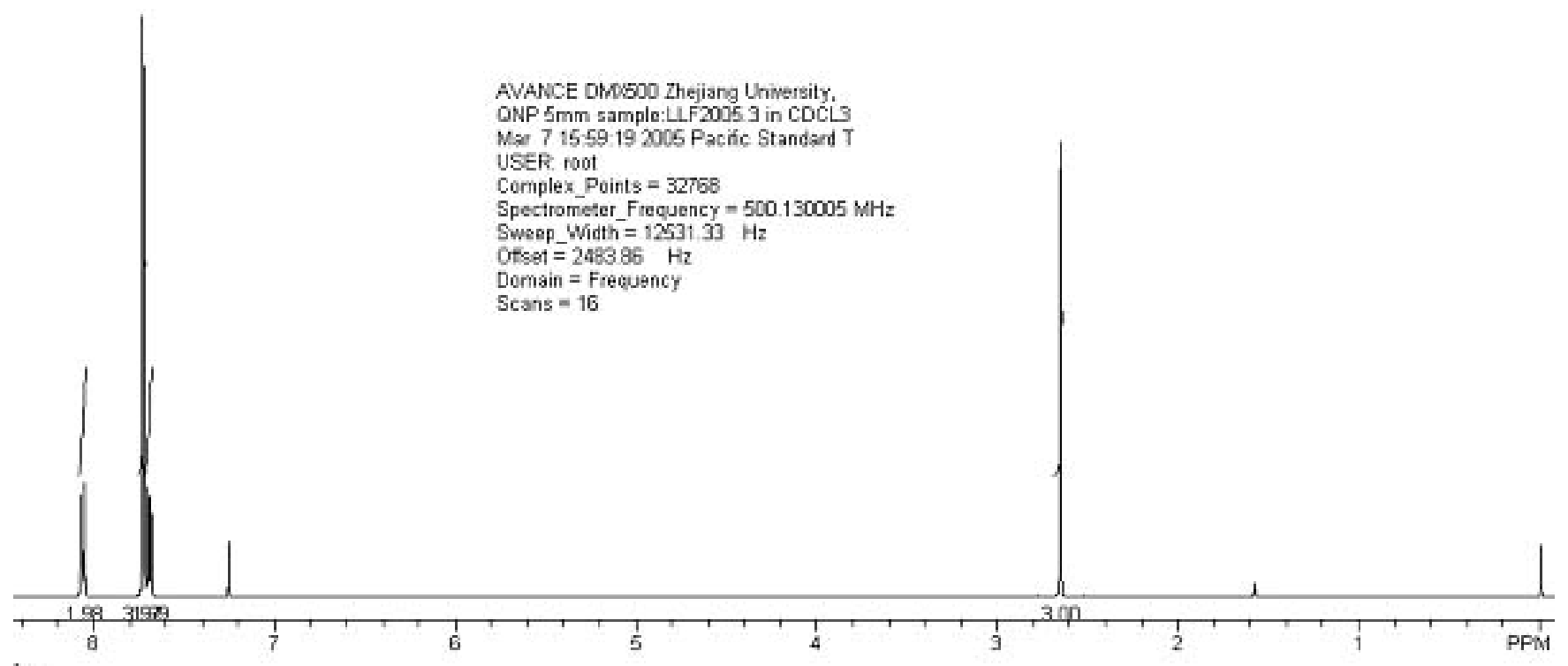




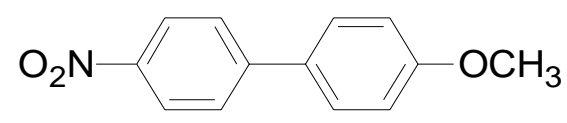

4'-Methoxy-4-nitro-biphenyl [2143-90-0, ref 12, T3-11]

${ }^{1} \mathrm{H}$ NMR (500 MHz, $\mathrm{CDCl}_{3}$, TMS): $\delta 8.28(\mathrm{~d}, 2 \mathrm{H}, J=9.0 \mathrm{~Hz}), 7.71(\mathrm{~d}, 1 \mathrm{H}, J=16.1 \mathrm{~Hz}), 7.69$ (d, $2 \mathrm{H}$, $J=8.5 \mathrm{~Hz}), 7.59(\mathrm{~d}, 2 \mathrm{H}, J=8.5 \mathrm{~Hz}), 7.02(\mathrm{~d}, 2 \mathrm{H}, J=9.0 \mathrm{~Hz}), 3.88(\mathrm{~s}, 3 \mathrm{H}) . \mathrm{MS}(\mathrm{EI}): \mathrm{m} / \mathrm{z}(\%): 229$ (100) $\left[\mathrm{M}^{+}\right], 199$ (17), 183 (12), $152(13), 139$ (35), 128 (6), 63 (4).
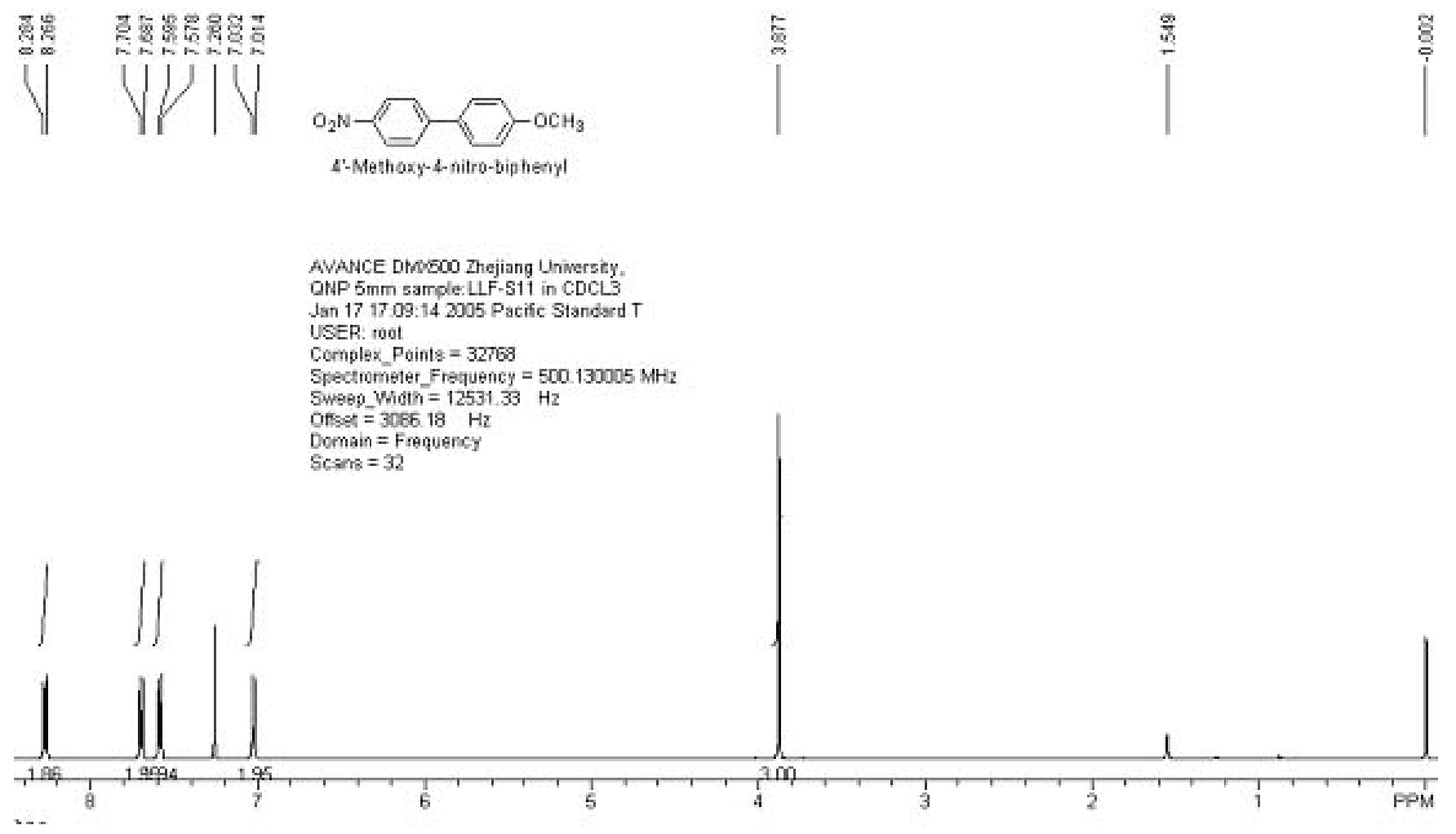


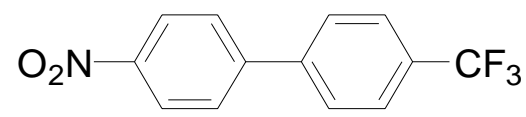

4-Nitro-4'-trifluoromethyl-biphenyl [80245-34-7, ref 13, T3-12]

${ }^{1} \mathrm{H}$ NMR (500 MHz, $\mathrm{CDCl}_{3}$, TMS): $\delta 8.34$ (q, $\left.2 \mathrm{H}, J=2.9 \mathrm{~Hz}\right), 7.76$ (m, $\left.6 \mathrm{H}\right) . \mathrm{MS}(\mathrm{EI}): \mathrm{m} / \mathrm{z}(\%): 267$

(100) $\left[\mathrm{M}^{+}\right], 248$ (10), 237 (40), 209 (33), 201(50), 170 (7), 152(51) ,75(4).
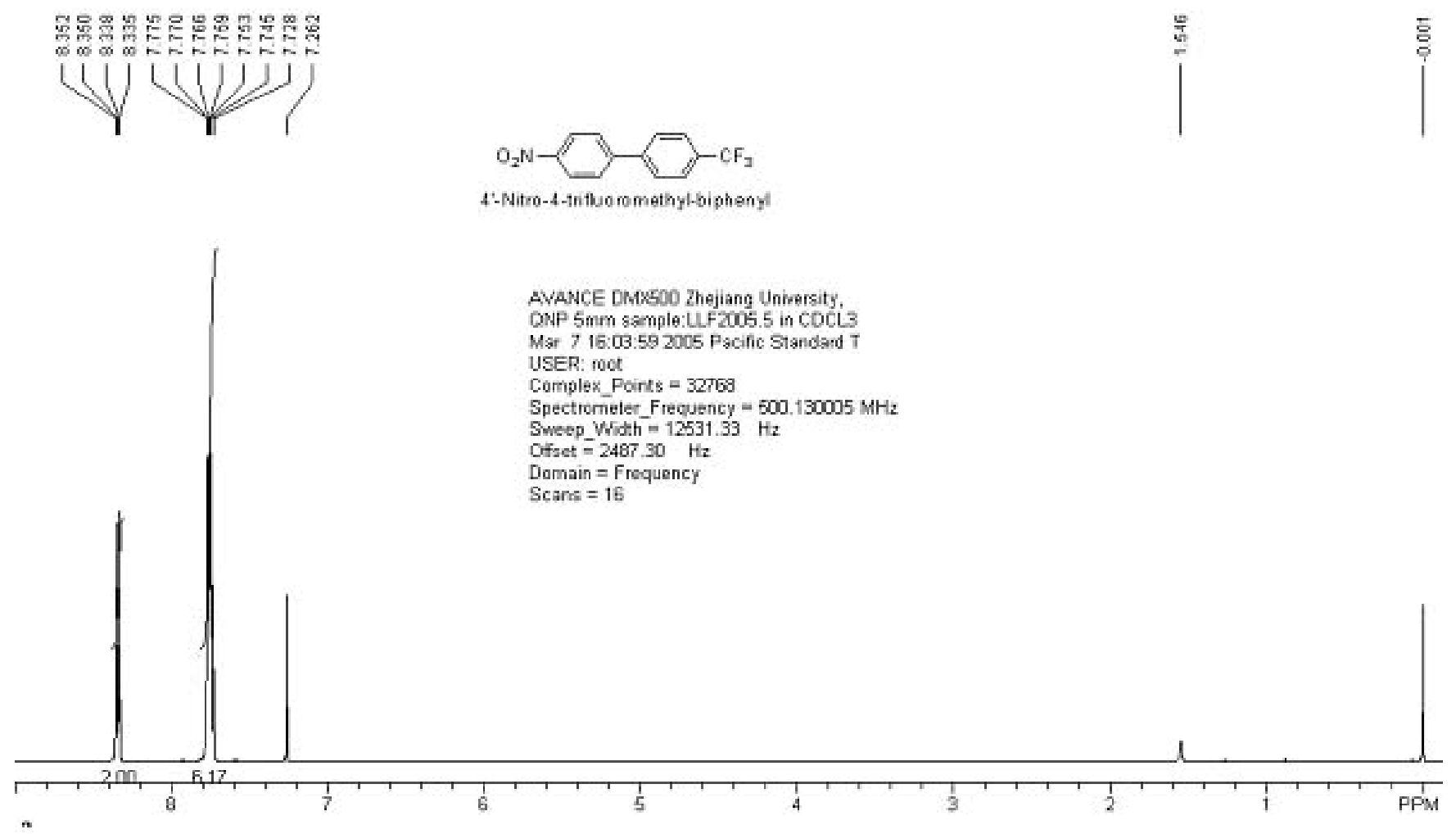
Reference

1. Tao, B.; Boykin, D.W. J.Org. Chem. 2004, 69, 4330-4335.

2. Sakurai, H.;Tsukuda, T.; Hirao, T. J.Org. Chem. 2002, 67, 2721-2722.

3. Dupuis, C.; Adiey, K.; Charruault, L.; Michelet, V.; Savignac, M.; Genet, J.-P. Tetrahedron Lett. 2001, 42, 6523-6526.

4. Leadbeater, N. E.; Marco, M. Org. Lett. 2002, 4, 2973-2976.

5. Xiong, Z.; Wang, N.; Dai, M.; Li ,A.; Chen, J.; Yang, Z. Org. Lett. 2004, 6, 3337-3340.

6. Adjabeng, G.; Brenstrum ,T.; Wilson, J.; Frapton, C.; Robertson, A.; Hillhouse, J.; McNulty, J.; Capretta, A. Org. Lett. 2003, 5, 953-955.

7. Wallow, T. I.; Novak, B. M. J.Org. Chem. 1994, 59, 5034-5037.

8. Hennings, D. D.; Iwama, T.; Rawal. V. H. Org. Lett. 1999, 1, 1205-1208.

9. Navarro, O.; Kaur, H.; Mahjoor, P; Nolan, S. P. J.Org. Chem. 2003, 69, 3173-3180

10. Indolese, A. F. Tetrahedron Lett. 1997, 38, 3513-3516.

11. Farina, V.; Krishnan, B.; Marshall, D. R.; Roth, G. P. J.Org. Chem. 1993, 58, 5434-5444.

12. Kataoka, N.; Shelby, Q.; Stambuli, J. P.; Hartwig, J. F. J.Org. Chem. 2002, 67, 5553-5566..

13. Marhold, A.; Klauke, E. Journal of Fluorine Chemistry, 1981, 18, 281-91. 